\title{
Efficient Shadow Removal Using Subregion Matching Illumination Transfer
}

\author{
Chunxia Xiao ${ }^{\dagger 1}$ Donglin $\mathrm{Xiao}^{1}$ Ling Zhang $^{1}$ and Lin Chen ${ }^{1}$ \\ ${ }^{1}$ School of Computer, Wuhan University, Wuhan, China
}

\begin{abstract}
This paper proposes a new shadow removal approach for input single natural image by using subregion matching illumination transfer. We first propose an effective and automatic shadow detection algorithm incorporating global successive thresholding scheme and local boundary refinement. Then we present a novel shadow removal algorithm by performing illumination transfer on the matched subregion pairs between the shadow regions and non-shadow regions, and this method can process complex images with different kinds of shadowed texture regions and illumination conditions. In addition, we develop an efficient shadow boundary processing method by using alpha matte interpolation, which produces seamless transition between the shadow and non-shadow regions. Experimental results demonstrate the capabilities of our algorithm in both the shadow removal quality and performance.
\end{abstract}

Categories and Subject Descriptors (according to ACM CCS): I.3.3 [Computer Graphics]: Picture/Image Generation-Line and curve generation

\section{Introduction}

Shadow detection and removal is fundamental problem in computer graphics and computer vision communities, which can greatly improve the performance of various applications, such as image interpretation and object classification. Shadow removal is also beneficial to increase the visual realism and physical realism in image editing and processing. Many shadow removal approaches have been proposed in the last decade (see [AHO11] for a survey). Although impressive results have been produced, as shadow removal is a difficult problem, these methods usually give assumption$\mathrm{s}$ under which these methods can be performed successfully. These assumptions include lighting conditions, image acquisition devices, surface materials, object reflectance, etc. Consequently, most methods are capable of producing good results for the images in which the assumptions hold.

In this paper, we propose a novel shadow removal method for the input single image. The shadow removal process consists of two challenging subtasks: shadow detection and

$\bar{\dagger}$ Corresponding to Chunxia Xiao: cxxiao@whu.edu.cn shadow removal. As a preprocess procedure, we first present an automatic shadow detection method to obtain accurate shadow boundaries. Then based on the shadow detection results, we develop an efficient shadow removal method using subregion matching illumination transfer to produce highquality shadow-free image. Note that as shadow removal is a very challenging problem, our method also has limitation in processing all kinds of shadow situation, however, we hope that the proposed method can provide an alternative way in shadow removal communities.

Many methods have been proposed for shadow detection. For example, Finlayson et al.[FHLD06] compared edges in the original RGB image to edges found in an illuminantinvariant image, and detected the shadows in the image automatically. As accurate shadow detection is an extremely difficult task, to detect complex shadows, user interaction also has been applied [WTBS07, SL08] to improve the shadow detection result. In this paper, we present a shadow detection method by exploiting a coarse-to-fine strategy. We first detect the coarse shadow regions by using global successive thresholding scheme [CLH09, Ots75], and then we improve the detected shadows using local guided filtering operator to 
refine the shadow boundaries, this method works well for our shadow removal procedure.

For an input image, the illumination conditions are usually complex, and the different materials usually have different reflectance (albedo) to the illumination, which make the shadows usually nonuniform. Thus, global uniform shadow removal method without considering the material and texture variation may not produce satisfactory results. For some complex shadow images, when the shadowed regions contain two or more kinds of textures or materials, handling each kind of regions separately is a better choice. Based on above observations, we propose a novel shadow removal algorithm by performing adaptive illumination transfer techniques [XSXM13] on the matched subregion pairs. First, we divide the detected shadow and non-shadow areas into different subregions based on texture segmentation, such that each subregion has similar textures or materials. Then, for each shadowed subregion, we find a corresponding nonshadowed subregion based on texture similarity matching. Finally, we perform adaptive illumination transfer method [XSXM13] on the matched pair of shadow and non-shadow subregions, and obtain the final shadow-free image.

Shadow boundary is the transition zone between the shadow areas and non-shadow areas, however, shadow removal around the shadow boundary is a challenging problem for the following reasons: (1) The shadow boundary area usually can not be accurately detected out, especially for complex shadow; (2) the illumination usually changes dramatically in the boundary regions, which makes it difficult to design an effective shadow removal algorithm on these regions. Several shadow boundary processing have been proposed [GDH11, SL08], however, there still are much space left to improve the affectivity and efficiency of shadow boundary removal. In this paper, we exploit the image alpha matte interpolation in shadow transition, and propose a new boundary processing method which can efficiently remove the shadow along the boundaries.

The main contributions of our work are as follows:

- Present a fast and effective shadow detection algorith$\mathrm{m}$ incorporating global successive thresholding scheme [CLH09] and local boundary refinement.

- Propose a shadow removal method based on subregion matching, which can process complex shadowed region$\mathrm{s}$ with different kinds of texture regions and illumination conditions.

- Develop an efficient shadow boundary processing method by using smooth alpha matte interpolation in computing shadow transition parameter.

In Figure 1, we give the overview for the proposed shadow removal system. For an input image, we first detect the shadow regions of the image, and utilize a guided filter to compute more accurate shadow boundaries. Then we perform shadow removal based on the subregion matching. Finally, we remove the shadow around the shadow boundaries and produce final shadow-free image. The various steps of the process are detailed in the following sections.

\section{Related Work}

Many shadow detection and shadow removal methods have been proposed, the comprehensive survey is beyond the cope of this paper, we only refer to the most related work to our paper.

Shadow detection: Many shadow detection approaches based on physical illumination model and color model have been proposed. For example, Finlayson et al.[FHLD06, FDL04, FHD02] transformed a color image into an illuminant invariance gray-scale image, which is related to the photographic function of camera and the reflection character of surface. Utilizing the illuminant invariance image, this method can automatically detect the shadow and has low time complexity. However, it may be difficult to get accurate shadow boundary for complex shadow images. Several data-driven approaches have been proposed that learn to detect shadows based on training images [ZSMT10, LEN10, GDH11]. For example, Zhu et al. [ZSMT10] proposed a learning-based approach for automatically detecting shadows in a single monochromatic image. Different from the traditional method utilizing pixel or boundary information, Guo et al. [GDH11] presented a shadow detection method using a relational graph of paired regions. Although visually pleasing results have been obtained, the time complexity of this method is relatively much higher. More automatic shadow detection algorithms refer to the survey paper [AHO11].

As automatic shadow detection is an extremely difficult task, for example, it is difficult to determine whether a pixel is dark due to the shadow or the reflectance at the corresponding scene point. Sometimes, even human being is difficult to accurately identify the shadowed regions from the image. Thus, user interaction also has been applied [WTBS07, SL08, LG08] improve the shadow detection result. However, for complex images, shadow detection is still a challenging problem, especially for processing high resolution images. For remote sensing images, where there are too much shadowed region, thus, much user interaction is tedious for this application.

Shadow removal: Finlayson and his colleagues proposed a series of shadow removal methods [FHLD06, FHD02, FDL04]. They treated shadow removal as a reintegration problem based on detected shadow edges. These shadow removal methods are good in the theory formulation and achieve some impressive results. However, the reintegration method depends on precise detection of shadow edges, and inaccurate shadow edges may produce unpleasing results. Furthermore, these methods do not consider the effect of the ambient illumination and the variation of the object materials, thus, the fine texture detail 


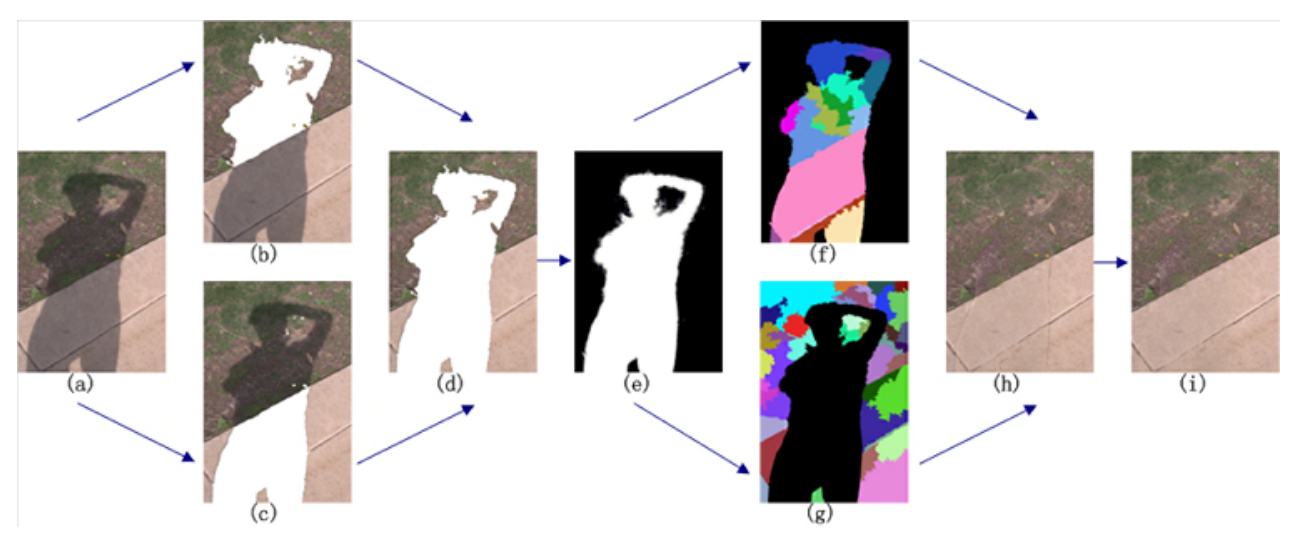

Figure 1: Overview of the proposed system. (a) Input image, $(b)(c)$ partial shadow detection result, $(d)$ coarse shadow detection result, $(e)$ accurate shadow detection result, $(f)(g)$ texture segmentation results, $(h)$ shadow removal result without boundary handling, (i) shadow-free image.

in the shadow regions can not be efficiently recovered. Liu et al. [LG08] presented a shadow removal approach preserving texture consistency between the original shadow and lit area. Unlike Finlayson's method, this method assigns gradients for the shadow and lit area according to illumination and gradient change in penumbra area. Similar to [FHLD06], Liu et al. [LG08] also recovered the shadow free image for the shadow area by solving a reintegration equation (Poisson equation). It is well known that solving the reintegration method (Poisson equation) is time-consuming for high resolution image, and these methods are also not convenient to process image with many disconnected shadow regions.

Inspired by color transfer technique [RAGS01], by building linear mapping models from the shadowed and lit areas, Shor et al. [SL08] described a method for producing a high-quality shadow-free image from a single input image, followed by a pyramid-based restoration process. However, this method does not work well for recover texture detail of the shadowed regions. More recently, improving the work [SL08, RAGS01], Xiao et al. [XSXM13] proposed an adaptive multi-scale illumination transfer technique, considering the material reflectance variation, this method not only effectively removes the shadows but also recovers the texture detail of the shadowed regions. Guo et al. [GDH11] calculated the ratio between direct light and environment light and obtained the recovered image by relighting each pixel. However, this method also does not take the reflectance variation into account, and it also can not recover the texture detail well. Arbel and Hel-Or [AHO11] considered each image channel as an intensity surface, and approximated the shape of the intensity surface in shadow regions to aid in obtaining shadow-free images. They also performed shadow-free region enhancement to further improve the quality of the final shadow-free image.

Shadow matting and editing: Chuang et al. [CGC*03] in- troduced a shadow matting and compositing equation which considered the input image as a linear combination of a shadow-free image and a shadowed image. Both [WT05] and [WTBS07] applied user-specified quadmap to simplify the shadow extraction and matting. Wu and Tang [WT05] formulated the shadow extraction problem into a Bayesian framework. Wu et al. [WTBS07] first computed an approximate shadowless image based on color transfer techniques [RAGS01], and then they used the shadowless image to define an optimization function to receive the final shadow matting results. Both [WT05] and [WTBS07] focus on shadow matte extraction, to process complex shadow scenes, these two methods still may not work well for shadow removal, especially recover the image detail in the shadow areas. Mohan et al. [MTC07] proposed a shadow editing tool by fitting a gradient domain shadow edge model to the shadows. Besides enabling users to remove the shadows, this method also can simulate a variety of lighting conditions, such as ambient-only lighting, for shadow editing.

\section{Shadow detection}

The HSI color model, which follows the human visual perception closely, separates the color components in terms of intensity, hue and saturation. Tsai [Tsa06] utilized the ratio value of the hue over the intensity to construct a ratio map for detecting shadows of color aerial images. The ratio map is built on the HSI color model. It has been observed that shadow regions usually have lower intensity value and higher hue value compared with other non-shadow regions in the image [Tsa06]. To highlight the difference between shadow regions and lit regions, inspired by [Tsa06], we construct a ratio map as follows: $r(x)=H(x) /(I(x)+0.01)$, where $I(x)$ and $H(x)$ refer to value of intensity and hue of the pixel $x$ in the input image, respectively. We apply the bilateral filter [TM98] to filter the input image to alleviate the noise effec- 
$\mathrm{t}$ before computing the ratio map $r(x)$. Note that we bound $r(x)$ to $[0,255]$ to make it more convenient for subsequent processing.

Based on the ratio map $r(x)$, we use the Otsu's method [Ots75] to compute a coarse shadow detection map $s$ which is consisted of candidate shadow regions and candidate nonshadow regions. The Otsu's method [Ots75] assumes that the image to be thresholded contains two classes of pixels, and calculates the optimum threshold separating those two classes so that their inter-class variance is maximum. We apply the Otsu's method over the histogram of the ratio map $r(x)$ to automatically select an optimal threshold $T$. The optimal threshold $T$ is determined as follows:

$$
T=\operatorname{argmax}\left\{w_{0}(T)\left(\mu_{0}(T)-\bar{\mu}\right)^{2}+w_{1}(T)\left(\mu_{1}(T)-\bar{u}\right)^{2}\right\}
$$

where $w_{0}(T)=\sum_{i=0}^{T} p_{i}, w_{1}(T)=\sum_{i=T+1}^{255} p_{i}, \bar{\mu}=\sum_{i=0}^{255} i \cdot p_{i}$, $\mu_{0}=\sum_{i=0}^{T} i \cdot p_{i}, \mu_{1}=\sum_{i=T+1}^{255} i \cdot p_{i}$ and $p_{i}$ is the probability of the gray level $i$. According to the threshold $T$, a coarse shadow detection map $s$ can be obtained by:

$$
s(x)= \begin{cases}1, & r(x)>T \\ 0, & \text { otherwise }\end{cases}
$$

here, $s(x)=1$ refers to those pixels lying in the candidate shadow regions in the image, and $s(x)=0$ refers to those pixels lying in the candidate non-shadow regions. Instead of only using the global thresholding process in Tsai's algorith$\mathrm{m}$, we also apply the successive thresholding scheme (STS) [CLH09] to detect shadows more accurately.

This method produces satisfactory results for remote sensing images, when applied for nature images, this method needs to be further improved. Since for most natural images, the illumination is usually complex, and the object materials and textures also vary greatly. Sometimes, not all the shadow areas can be detected out for images with various texture types using only once detection processing. For example, the image in Figure 1 has three kinds of textures (grass, soil and pavement), and only the grass and the soil are identified using above detection procedure for once. To detect the shadows from the image with several kinds of textures, we usually repeat the above procedure for two or three times, depending on the image complexity. Figure 1(d) shows an final detection result using shadow detection process twice.

The result produced by this method is binary shadow detection result, to receive more accurate shadow boundary detection results. We apply the guided filter [HST10] to refine the shadow boundary detection. Let $I$ be the original image which acts as the guidance image, the binary shadow image $s$ is the input image, the filer output is $q$, the $\omega_{k}$ is local window centered at the pixel $k$. The guided filter is a local linear model between the guidance $I$ and the filter output $q$ $: q_{x}=a_{k}^{T} I_{x}+c_{k}, \forall x \in \omega_{k}$, where $a_{k}$ and $c_{k}$ are the filtering coefficients [HST10]. By applying Integral Image technique [Cro84], the filter can be computed in $O(N)$ time.With the guided filter, the input binary shadow mask is refined to be the alpha matte near the binary shadow boundary. We then truncate the alpha matte $q$ according to given threshold, and obtain the final shadow result $S$. As illustrated in Figure 2, with the original image as the guidance, the refined shadow detection result is more smooth and accurate than the original shadow mask, especially at the shadow boundaries. The alpha matte $q$ will also be used in the following boundary shadow processing.

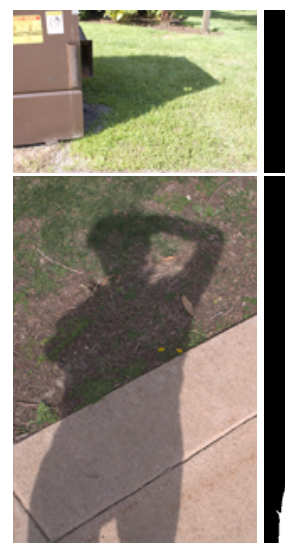

(a)

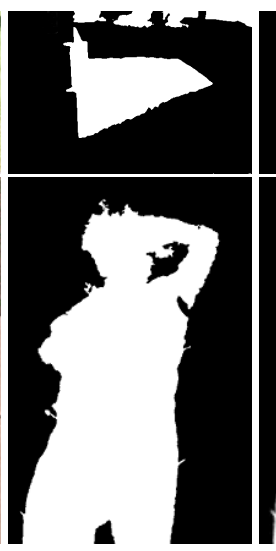

(b) (c)

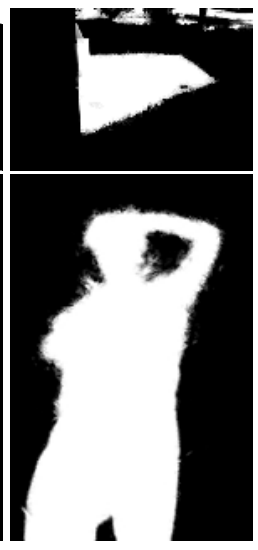

Figure 2: Shadow detection: (a) Input image, (b) binary shadow detection result, (c) refined shadow detection result.

\section{Shadow removal using subregion matching}

For complex images with different object materials and illumination conditions, to derive satisfactory shadow removal results, the selection of the lit regions is important. As shown in Figure 3, the texture materials of the wood blocks are complex, and the illumination conditions are also varying, there are umbra, penumbra and other complex soft shadows in the images. If we apply the uniform illumination transfer technique [SL08, XSXM13], and transfer the illumination information of one lit region to all shadow regions to perform shadow removal, the results are not convincing. Thus, in these complex cases, to receive satisfactory results, for the shadow regions with different textures, materials and illumination conditions, we have to select appropriate lit regions as samples to produce better results. In the following sections, we develop a shadow removal methods based on subregion matching.

\subsection{Texture segmentation and matching}

We first segment the shadowed regions and lit regions into subregions, respectively, and each subregion has similar texture. In our system, we apply the graph-based image segmentation [FH04] to perform the image segmentation process, where the image pixels with similar material and texture are clustered together. As illustrated in Figure 4, in the 


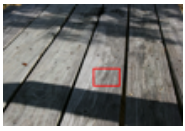

(a)

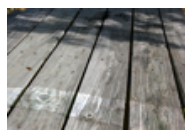

(b)

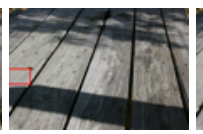

(c)

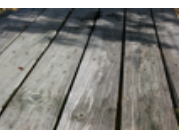

(d)
Figure 3: Limitation of shadow removal method using global uniform illumination transfer. (a) and (c) are the selected different lit regions on the input image. $(b)(d)$ are corresponding shadow removal results based on the selected lit regions $(a)$ and $(c)$, respectively.

shadow regions, even in the same wood block, the shadow regions may have different materials and illumination. Each block is segmented in several subregions, and each subregion has similar color distribution and texture structure. The lit regions are segmented in the similar way.
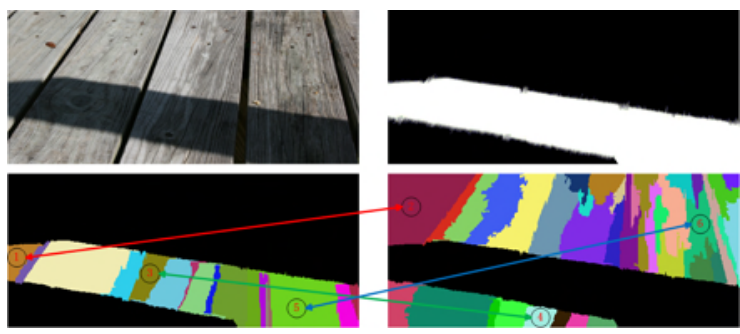

Figure 4: Texture clustering. Top left: Input image, top right: detected shadow, bottom left: texture clustering results of shadowed regions, bottom right: texture segmentation result$s$ of lit regions. The corresponding subregion pairs are also illustrated in bottom left and bottom right.

For each subregion in shadowed region, we find a corresponding subregion in the lit regions based on the subregion matching. The subregion matching operator is consisted of two steps. We first extract the textural feature for each subregion of both the shadowed and lit regions. We apply the feature representation based on Gabor wavelet transfor$\mathrm{m}$ [MM96] to extract the texture feature. Then we perform the subregion matching procedure, for each subregion in the shadow regions, we find a best matching subregion in the lit regions, and obtain the subregion matching pairs. Finally, for each subregion matching pair, we apply the adaptive illumination transfer technique to complete the shadow removal.

Let $S=\left\{s_{1}, s_{2}, \cdots, s_{N}\right\}$ and $B=\left\{b_{1}, b_{2}, \cdots, b_{M}\right\}$ be the set of subregions inside the shadow areas and the lit areas, respectively. $N$ is the number of the subregions in $S$, and $M$ is the number of subregions in $B$. Subregion matching is performed in the following two steps:

Step 1: Texture feature computing. Compute the texture features for each subregion of $S$ and $B$. According to [MM96], we compute the feature vector $\bar{f}=$ $\left[\mu_{00} \sigma_{00} \mu_{01} \sigma_{01} \cdots \mu_{m n} \sigma_{m n}\right](0 \leq m<4,0 \leq n<6)$ for each subregion. The $\mu_{m n}$ and $\sigma_{m n}$ represent the mean and the standard deviation of the magnitude of the transform coefficients $w_{m, n}$, which is the Gabor wavelet transform of subregion, and subscripts $m$ and $n$ are the index of the scale and orientation, respectively. The feature vector $\bar{f}$ represents the local structures of the subregion in multiple scales and orientations. We denote $\bar{f}_{s}^{(i)}$ and $\bar{f}_{b}^{(j)}$ the corresponding feature vectors for $s_{i}$ and $b_{j}$.

Step 2: Subregion matching. For patch $s_{i}$ and $b_{j}$, our patch matching method considers two kinds of metrics: the texture feature distance dist_texture $(i, j)$ and the space distance dist_space $(i, j)$. More especially, the texture feature distance measures the difference between two corresponding subregions in the texture feature space:

$$
\text { dist_texture }(i, j)=\sum_{m} \sum_{n} d_{m n}(i, j)
$$

$$
d_{m n}(i, j)=\left|\frac{\mu_{m n}^{(i)}-\mu_{m n}^{(j)}}{\alpha\left(\mu_{m n}\right)}\right|+\left|\frac{\sigma_{m n}^{(i)}-\sigma_{m n}^{(j)}}{\alpha\left(\sigma_{m n}\right)}\right|
$$

$\mu_{m n}^{(i)}\left(\right.$ or $\left.\mu_{m n}^{(j)}\right)$ and $\sigma_{m n}^{(i)}\left(\right.$ or $\sigma_{m n}^{(j)}$ ) are the mean and the standard deviation of texture features of subregion $s_{i}$ and $b_{j}$, respectively. $\alpha\left(\mu_{m n}\right)$ and $\alpha\left(\sigma_{m n}\right)$ are the mean and the standard deviation of the magnitude of $w_{m, n}$ computed in the whole input image $I$.

The space distance between the two subregions $s_{i}$ and $b_{j}$ is defined as:

$$
\text { dist_space }(i, j)=\sqrt{\left(\bar{X}^{(i)}-\bar{X}^{(j)}\right)^{2}+\left(\bar{Y}^{(i)}-\bar{Y}^{(j)}\right)^{2}}
$$

where $\bar{X}^{(i)}\left(\right.$ or $\left.\bar{X}^{(j)}\right)$ and $\bar{Y}^{(i)}\left(\right.$ or $\left.\bar{Y}^{(j)}\right)$ indicate the mean value of the x-coordinate and y-coordinate of all the pixels in $s_{i}$ (or $b_{j}$ ).

In our experiments, for each subregion $s_{i}$, we find the most similar $N_{T}$ (in our experiments $N_{T}=10$ ) subregion$\mathrm{s}$ in $B$, sort these subregions in distance increasing order and save these indexes in array Mark_texture $\left[N_{T}\right]$. Similarly, for each subregion $s_{i}$, we extracting the $N_{S}$ (we set $N_{S}=5$ in our experiments) subregions in $B$ with minimum space distance to $s_{i}$, and save the indexes of these subregions in mark_space $\left[N_{S}\right]$. The subregion matching is performed as follows:

Rule 1: If there is only one subregion $b_{j}$ that is in both Mark_texture $\left[N_{T}\right]$ and Mark_space $\left[N_{S}\right]$, then $b_{j}$ is the best subregion that matches $s_{i}$.

Rule 2: If there are two or more subregion $b_{j}$ that lie in both Mark_texture $\left[N_{T}\right]$ and Mark_space $\left[N_{S}\right]$, then the subregion $b_{j}$ with minimum sum of the indexes in mark_texture $\left[N_{T}\right]$ and Mark_space $\left[N_{S}\right]$ is selected as the best matching subregion.

Rule 3: If there is no any subregion that lies in both these 
arrays, the region merged from all subregions in these two arrays, serves as the best-matching subregion for $s_{i}$.

Figure 4 show some subregion matching results, for each subregion in the shadowed regions, we find its best matching subregion in lit regions. For example, subregion 1, 2, 3 in the shadow regions matches subregion 4, 6, 5 in lit areas, respectively.

\subsection{Shadow removal}

In image formation equation [Bar], an image $I(x)$ at the pixel $x$ is the pixel wise product of illumination and reflectance:

$$
I(x)=R(x) L(x)
$$

where $L(x)$ and $R(x)$ are the illumination and the reflectance (albedo) at the pixel $x$.

Assuming a scene whose shadows are cast due to a single primary source of illumination, if a pixel $x$ is in lit region, the illumination can be described as a sum of two terms, $L(x)=L^{d}(x)+L^{a}(x)$, where $L^{d}(x)$ is the direct illumination and $L^{a}(x)$ is the indirect (ambient) illumination. Thus the intensity at pixel $x$ is : $I^{l i t}(x)=L^{d}(x) R(x)+L^{a}(x) R(x)$. If some object occludes the primary light source, it will cast a shadow at pixel $x$. As this occluder would also block some of the ambient illumination, thus, the reflected intensity at pixel $x$ is $I^{\text {shadow }}(x)=\eta(x) L^{a}(x) R(x)$, where $\eta(x)$ is the attenuation factor of the ambient illumination.

Based on above observation, Shor et al. [SL08] applied the illumination transfer approach [RAGS01] for shadow removal. The lit intensity at $x$ can be expressed as an affine function between the illuminated and shadowed intensities :

$$
I^{l i t}(x)=L^{d}(x) R(x)+\frac{1}{\eta(x)} I^{\text {shadow }}(x),
$$

This affine function can be reformulated as :

$$
I_{k}^{l i t}(x)=A_{k}^{a v g}(x)+\gamma(x) I_{k}^{\text {shadow }}(x),
$$

where $A_{k}^{a v g}=\mu_{k}(B)-\gamma \mu_{k}(S)(k \in\{R, G, B\})$ is the reflected direct illumination in the three RGB color channels, $\mu(S)$ and $\mu(B)$ are the mean color of the pixels in shadow regions $S$ and lit regions $B, \gamma(x)=\frac{1}{\eta(x)}=\frac{\sigma(B)}{\sigma(S)}$, and $\sigma(S)$ and $\sigma(B)$ are the standard deviation of their luminance.

More recently, Xiao et al.[XSXM13] presented an adaptive illumination transfer approach for shadow removal, and improved $A_{k}^{a v g}$ using adaptive parameter:

$$
A_{k}(x)=A_{k}^{a v g} \frac{I_{k}(x)}{I^{a v g}}
$$

where $I_{k}(x)$ is the intensity value for pixel $x$, and $I^{a v g}=\mu(S)$ is the mean color at the pixels in $S . A_{k}(x)$ varies according to the reflectance variation at pixel $x$, which efficiently derives a shadow-free image and avoids the loss of texture details.

Based on above discussion, for each matched subregion pair $s_{i}$ and $b_{j}$, we remove the shadow in $s_{i}$ using the adaptive illumination transfer approach [XSXM13]. Note that the shadow removal is performed in the Lab color space. After finishing the shadow removal processing, the results are transformed back to RGB image. As illustrated in Figure 5 , with the adaptive illumination transfer performed on the paired subregions, the shadows are better removed, and the shadow-free regions are more consistent with the surrounding scenes.

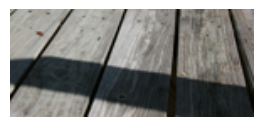

(a)

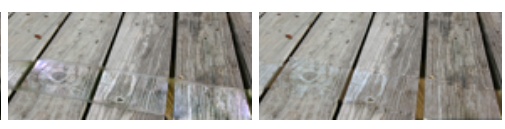

(b)

(c)

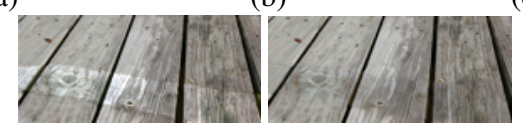

(d) (e)

Figure 5: Shadow removal comparison. (a)Input image, (b)result of Finlayson [FHLD06],(c)result of Shor [SL08], (d) result of Guo [GDH11],(e) our result.

\subsection{Shadow boundary processing}

As the illumination usually changes dramatically in the shadow boundary regions, shadow boundary processing is a challenging problem. In this section, we introduce new shadow boundary process method based on alpha matte interpolation to remove the shadows of the boundary. We obtain seamless and shadow-free image around the shadow boundaries, and efficiently recover the texture detail of the boundaries.

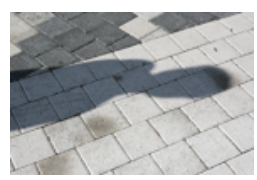

(a)

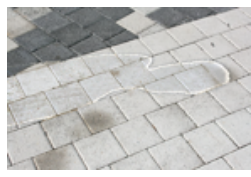

(b)

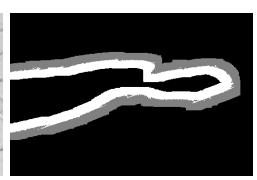

(c)

Figure 6: Shadow boundaries processing. (a) Input image, (b) shadow boundaries needed to be processed, (c) the narrow bands built around the shadow boundaries.

We apply the color and illumination information of the neighboring regions around the boundaries for computing the shadow-free image for the boundaries. As illustrated in Figure 6, we first specify narrow band $\phi_{S}$ (white narrow band) in the shadowed region neighboring to boundaries, and similarly, specify narrow band $\phi_{B}$ (gray narrow band) in the lit region neighboring to boundaries. The narrow band$\mathrm{s}$ can be computed using image morphological operations, such as dilation and erosion, around the boundaries. We 


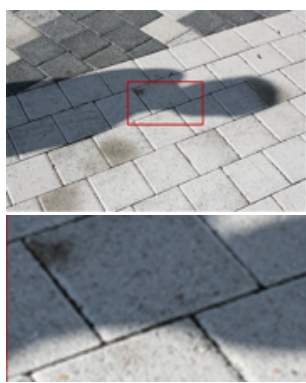

(a)

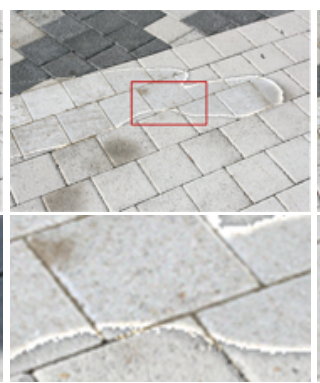

(b)

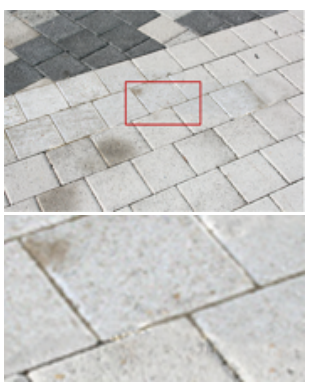

(c)

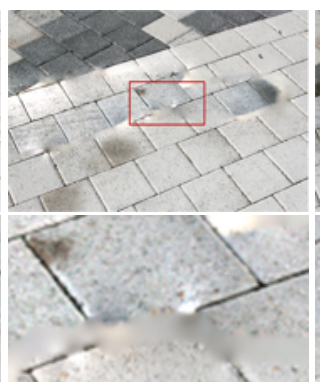

(d)

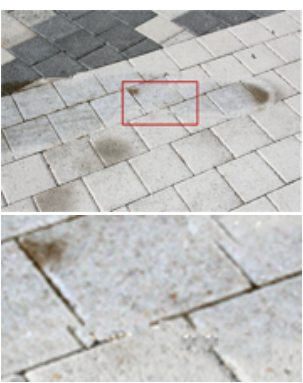

(e)

Figure 7: Boundary handling results. (a) Input image, (b)our result without shadow boundary handling, (c)our result with boundary handling, (d) shadow removal result of Finlayson et al.[FHLD06], (e)shadow removal result of Shor et al.[SL08]

then utilize the information in these two bands to infer the shadow-free image of the boundaries.

Chuang et al.[CGC*03] introduced a shadow composition equation, which expresses the observed image $I_{k}(x)$ as a linear combination of the lit image $B_{k}(x)$ and the shadowed image $S_{k}(x)$ :

$$
I_{k}(x)=\alpha S_{k}(x)+(1-\alpha) B_{k}(x)
$$

where $k \in\{R, G, B\}$, and $\alpha$ is the shadow matte value. By reforming Eq.10, we receive:

$$
\alpha(x)=\frac{I_{k}(x)-B_{k}(x)}{S_{k}(x)-B_{k}(x)}
$$

Following previous work [Bar], an image $I_{k}(x)$ is considered to be the wise product of illumination field $L(x)$ and reflectance $R(x), I_{k}(x)=L(x) R(x)$, and the illumination is composed of two terms: $L(x)=L^{d}(x)+L^{a}(x)$, where $L^{d}(x)$ is the direct illumination and $L^{a}(x)$ is the ambient illumination. With the primary light source occluded in the shadow area, in the shadowed regions, the color intensity is formed by the ambient illumination. The intensity of the shadow area $S_{k}(x)$ and the lit area $B_{k}(x)$ can be expressed as:

$$
\begin{gathered}
B_{k}(x)=\left(L^{d}(x)+L^{a}(x)\right) R(x) \\
S_{k}(x)=L^{a}(x) R(x)
\end{gathered}
$$

With above discussion, we have:

$$
\begin{aligned}
\alpha(x) & =\frac{\lambda_{k} A_{k}(x)-\left(L^{d}(x)+L^{a}(x)\right) R(x)}{L^{a}(x) R(x)-\left(L^{d}(x)+L^{a}(x)\right) R(x)} \\
& =\frac{\frac{\lambda_{k} A_{k}(x)}{L^{a}(x) R(x)}-\left(\frac{L^{d}(x)}{L^{a}(x)}+1\right)}{1-\left(\frac{L^{d}(x)}{L^{a}(x)}+1\right)}
\end{aligned}
$$

In the shadowed region, the intensity at pixel $x$ produced by ambient illumination $S_{k}(x)=L^{a}(x) R(x)$ can be approximated using the observed image $I_{k}(x)$. With the assumption of local smoothing, we have $S_{k}(x) \approx I_{k}^{\prime a v g}$, which $I_{k}^{\prime a v g}$ is the mean intensity value of the pixels in the window with radius $r$ centered at the pixel $x$ (we set $r=3$ in our experimentations). Since the bands bounding the shadow boundary is narrow, we consider $I_{k}^{\prime a v g} \approx I_{k}^{a v g}=\mu_{k}\left(\phi_{S}\right)$. Thus, with Eq.9, Eq.14 can be expressed as:

$$
\alpha(x) \approx \frac{(1+h)-\frac{\lambda_{k} A_{k}(x)}{I_{k}^{\text {avg }}}}{h}=\frac{(1+h)-\frac{A_{k}(x)}{A_{k}^{\text {agg }}}}{h}
$$

here $h$ is defined as:

$$
h=\frac{L^{d}}{L^{a}}=\frac{\bar{B}-\bar{S}}{\bar{S}}=\frac{\frac{1}{W_{B}} \sum p_{x}-\frac{1}{W_{S}} \sum q_{y}}{\frac{1}{W_{S}} \sum q_{y}}, x \in \phi_{B}, y \in \phi_{S}
$$

where $\bar{B}$ and $\bar{S}$ are the average luminance of lit and shadowed bands $\phi_{B}$ and $\phi_{S}, W_{B}$ and $W_{S}$ are the number of piexls in $\phi_{B}$ and $\phi_{S}$, respectively. $\bar{B}$ and $\bar{S}$ are computed at the $l$ channel, and $p_{x}$ and $q_{y}$ are the pixel in $\phi_{B}, \phi_{s}$ respectively.

From Eq.15, we can build the relationship between $\alpha(x)$ and $A_{k}(x)$. We compute the desirable $A_{k}(x)$ as follows:

$$
A_{k}(x) \approx A_{k}^{a v g}((1-\alpha(x)) h+1)
$$

where $A_{k}^{a v g}=\mu_{k}\left(\phi_{B}\right)-\gamma \mu_{k}\left(\phi_{S}\right)(k \in\{R, G, B\})$ is computed using the information of shadowed and lit band $\phi_{S}$ and $\phi_{B}$, and $\alpha(x)$ is computed using the guidance filtering described in section 3. With the computed $A_{k}(x)$, we apply the shadow removal model (Eq.8) to remove the shadow around the boundaries and receive image $I_{k}^{b f}(x)$. From the Eq.17, we observe that $A_{k}(x)$ transitions smoothly in the boundary regions according to the variation of $\alpha(x)$, which is consistent with the illumination variation.

To make a smoother transmission at the shadow boundary, we blend the boundary processing result $I_{k}^{b f}(x)$ with the input shadow image $I_{k}(x)$ using a blending function $p(x)$ and receive the final shadow-free image $I_{k}^{\text {free }}$ :

$$
I_{k}^{f r e e}=I_{k}^{b f}(x) \alpha(x)+I_{k}(x)(1-\alpha(x)) p(x)
$$

Here, the blending function $p(x)$ is setting as: $p(x)=$ $a \alpha(x)^{2}+b$, where $a, b$ are the parameters, and in our experiments, we set $a=0.2$, and $b=1.15$.

Note that in the shadow removal section, the shadow removal results around the boundaries between the subregions may not be illumination inconsistent. We also apply this 
shadow boundary processing method to produce global consistent illumination on the final shadow-free images.

Figure 7 shows our shadow boundary processing results. We also present comparison results with previous methods [FHLD06, SL08]. The results show that our method is better to recover the image details and is faithful to preserve original texture of original images.

\subsection{Algorithm overview}

In algorithm 1, we outline the main steps of the proposed shadow removal algorithm described in this paper.

\section{Algorithm 1: Shadow removal algorithm \\ Input: RGB image $I$ \\ Output: Shadow-free RGB image}

1. Shadow detection: Detect the shadow regions $S$ from $I$, the rest regions are lit regions $B$.

2. Texture segmentation: Segmenting $S, B$ into subregions $\left\{s_{1}, s_{2}, \cdots, s_{N}\right\},\left\{b_{1}, b_{2}, \cdots, b_{M}\right\}$, respectively.

3. Subregions matching: Find a optimal matching subregion $b_{j}$ in $B$ for each subregion $s_{i}$ in $S$.

4. Shadow removal: For the subregion pair $\left(s_{i}, b_{j}\right)$, remove the shadow of subregion $s_{i}$ applying the adaptive affine shadow formation model.

5. Boundary processing: Remove the boundary shadow of $S$ using alpha matte interpolation.

\section{Results and Discussion}

In this section, we show the results generated by our algorithm and compare our results to the most related methods. All our results are implemented using $\mathrm{C}++$ on a machine equipped with Pentium(R) Dual-Core CPU E5200@2.50GHz with 2GB RAM.

In Figure 7, we give the comparison results on the shadow boundaries processing. Shor et al. [SL08] processed the shadow boundary using graph-cut based texture inpainting [KSE* 03$]$. This method can produce visually pleasing results for images with abundant self-similar texture information, while for shadow boundaries with sharp structures, while this method cannot receive faithful shadow free boundaries since inpainting method will modify the image boundary content. The method [FHLD06] depends on precise detection of shadow edges, without careful parameter tuning, inaccurate shadow edges may produce unpleasing results. Our method can produce seamless shadow-free results for images with self-similar texture or sharp structures, and will not modify the image content.

Figure 8 compares our shadow detection method to ground truth and the methods generated by the methods [GDH11] and [SL08]. For the results of [GDH11], we apply the code presented by the authors on the website. We can see the sometimes this method is not robust, some shadow areas cannot be detected out, it is also time-consuming using graph based pairwise classification. We also compared the interactive shadow detection method of Shor et al. [SL08], and this method applies closed-form interpolation [LLW08] to perform shadow detection. The results are good, however, this method [SL08] incorporates user interaction, and furthermore, this method is also time-consuming for solving the closed-form optimization [LLW08]. Our method works well in shadow detection, furthermore, our method is automatic and fast. It takes about $37.85 \mathrm{~s}, 58.33 \mathrm{~s}, 2.14 \mathrm{~s}$ for [GDH11] and [SL08] and our method, respectively, to complete the shadow detection for the image with size of $640 \times 425$ in the first row of Figure 8.

In Figure 5 and Figure 9, we remove some complex shadows from the images and also compare with related methods [FHLD06, SL08, GDH11]. In these examples, there are different object materials in each image. As illustrated in Figure 5, the texture materials of the wood blocks are complex, our shadow removal method using subregion matching produces the best results. In the top row of Figure 9, the different regions of the wooden wall have different materials, and the illumination conditions are also nonuniform, the uniform shadow removal methods [FHLD06, SL08, GDH11] do not work well for this image. The bottom row of Figure 9 shows another example. The image consists of three kind$\mathrm{s}$ of texture, the grass, soil, and the concrete pavement, our shadow removal result is more consistent with surrounding scenes than other three methods. For a natural image with $800 \times 600$ pixels, the running time for our shadow removal our algorithm is about 2 to 3 seconds.

In Figure 10, we give more comparison with [FHLD06, SL08, GDH11], we also give the ground truth of some shadow free images. The input images contain several kinds of textures. Our results are comparable with the ground truth, the texture detail of the shadowed regions is recovered, and shadow-free regions are consistent with the surrounding scene in illumination intensity.

Limitations: Our shadow removing algorithm is based on the subregion matching between the shadow areas and non shadow areas. However, in case when the shadowed regions are too dark and the texture information is lost (as illustrated in Figure 11), or the texture information of shadow areas is very fuzzy, our method may fail to remove the shadows. Since in this case, for the subregions of the shadowed areas, we cannot find its corresponding subregions in the lit areas. Furthermore, even finding the corresponding subregion with similar texture in the lit regions, more sophisticated algorith$\mathrm{m}$ need to be developed to remove the shadows.

\section{Conclusions and future work}

In this paper, we have presented a novel shadow removal approach. We first introduced a new shadow removal method 


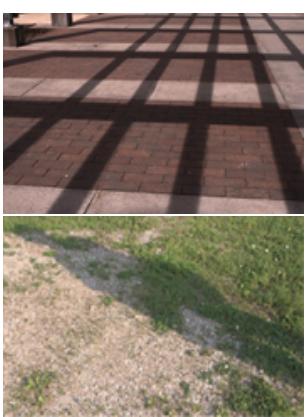

(a)

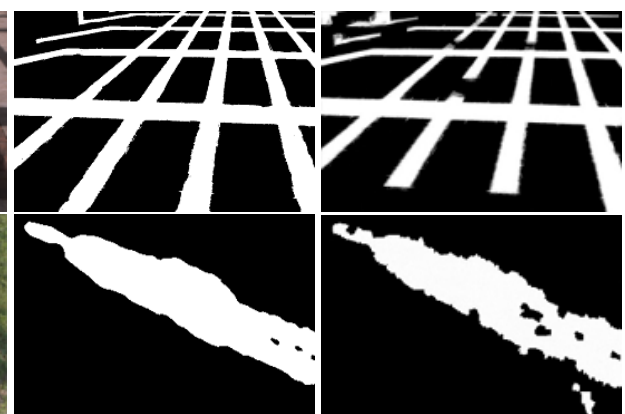

(b)

(c)

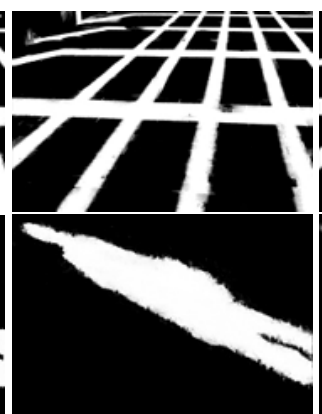

(d)

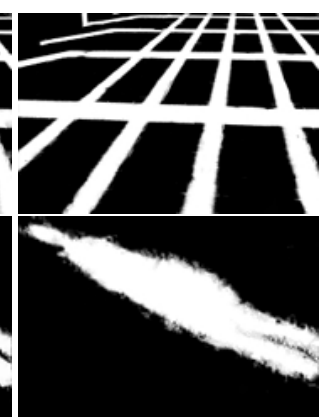

(e)

Figure 8: (a) Input image, (b) ground truth, (c) results of Guo et al.[FHLD06], (d) results of Shor et al. [SL08], (e) our results.

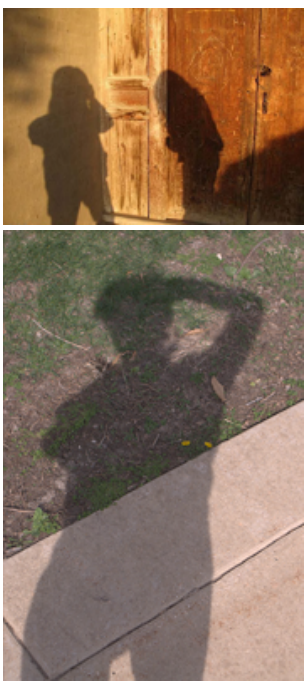

(a)

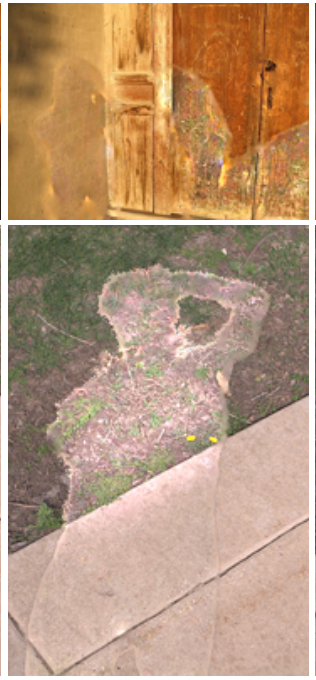

(b)

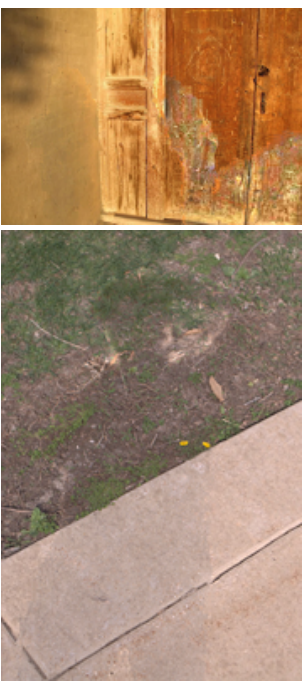

(c)

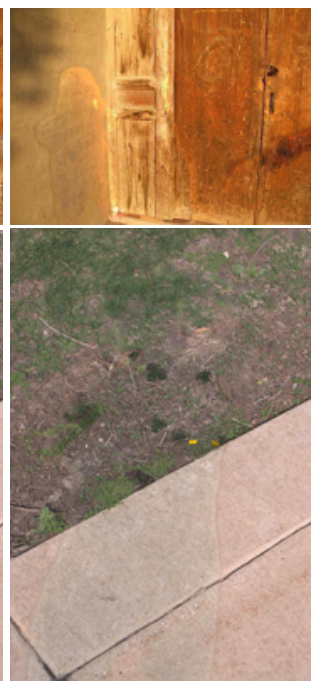

(d)

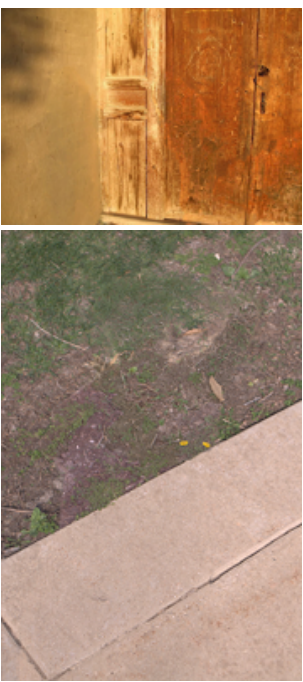

(e)

Figure 9: Shadow removal comparison. (a) Input images, (b) results of Finlayson[FHLD06], (c) results of Shor [SL08], (d) results of Guo [GDH11], (e) our results.

using texture matching, which can process shadowed image with non-uniform illumination and different texture materials. We also proposed a new shadow boundary processing method using alpha matte interpolation which produces shadow-free and seamless result. Our method can efficiently recover the texture detail of the shadowed regions, and will not destroy the content of the shadowed regions. In the future, we would like to extend our method to video shadow detection and removal. We are also interested in applying shadow removal in image dehazing [XG12] to produce more visually pleasing results.

\section{Acknowledgment}

This work was partly supported by the National Basic Research Program of China (No. 2012CB725303), the NSFC (No. 61070081, and No. 41271431), the Open Project Pro- gram of the State Key Lab of CAD\&CG (GrantNo. A1208), the Luojia Outstanding Young Scholar Program of Wuhan University, the Project of the Science and Technology Plan for Zhejiang Province (Grant No.2012C21004).

\section{References}

[AHO11] ARbEl E., Hel-Or H.: Shadow removal using intensity surfaces and texture anchor points. PAMI, IEEE Transactions on 33, 6 (2011), 1202-1216. 1, 2, 3

[Bar] BARROW H.: Recovering intrinsic scene characteristics from images. Computer vision systems. 6,7

[CGC*03] Chuang Y.-Y., Goldman D. B., Curless B., SALESIN D. H., SZELISKI R.: Shadow matting and compositing. In TOG (2003), vol. 22, ACM, pp. 494-500. 3, 7

[CLH09] Chung K.-L., Lin Y.-R., HuAng Y.-H.: Efficien$\mathrm{t}$ shadow detection of color aerial images based on successive 


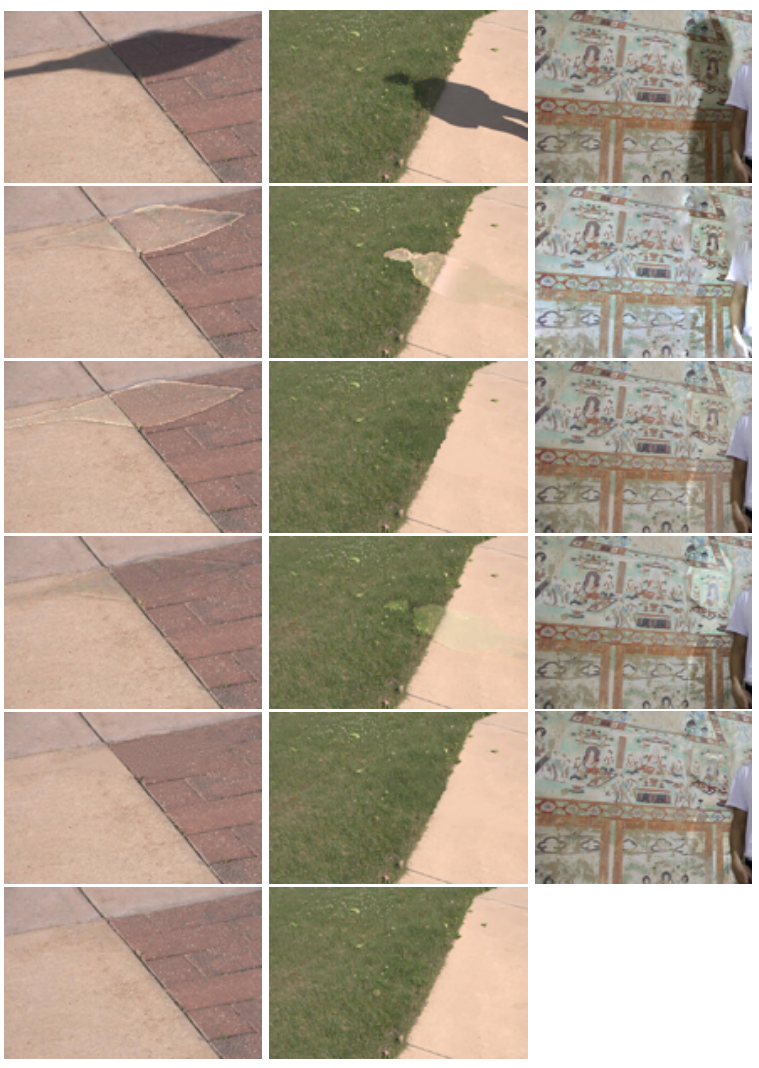

Figure 10: Shadow removal results. 1st row: input images, 2st row: results of Finlayson[FHLD06], 3st row: results of Shor[SL08], 4st row: results of Guo[GDH11], 5st row: our results, 6st row: groundtruth,

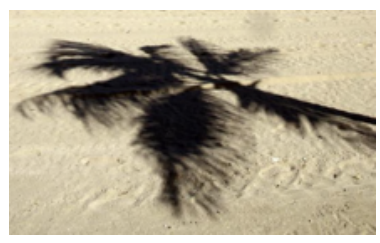

(a)

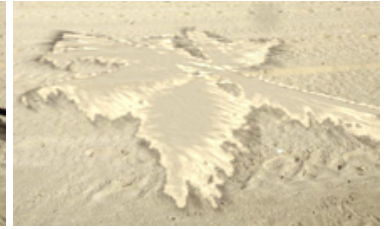

(b)
Figure 11: Limitation. (a) Input image, (b) our shadow removal result.

thresholding scheme. Geoscience and Remote Sensing, IEEE Transactions on 47, 2 (2009), 671-682. 1, 2, 4

[Cro84] CROw F. C.: Summed-area tables for texture mapping. In ACM SIGGRAPH Computer Graphics (1984), vol. 18, ACM, pp. 207-212. 4

[FDL04] Finlayson G. D., Drew M. S., Lu C.: Intrinsic images by entropy minimization. In ECCV 2004. Springer, 2004, pp. 582-595. 2

[FH04] Felzenszwalb P. F., Huttenlocher D. P.: Efficient graph-based image segmentation. International Journal of Computer Vision 59, 2 (2004), 167-181. 4

[FHD02] Finlayson G. D., Hordley S. D., Drew M. S.: Removing shadows from images. Lecture Notes in Computer Science (2002), 823-836. 2

[FHLD06] Finlayson G. D., HORDley S. D., Lu C., DREW M. S.: On the removal of shadows from images. PAMI 28, 1 (2006), 59-68. 1, 2, 3, 6, 7, 8, 9, 10

[GDH11] Guo R., DAI Q., HoIEM D.: Single-image shadow detection and removal using paired regions. In CVPR 2011 (2011), IEEE, pp. 2033-2040. 2, 3, 6, 8, 9, 10

[HST10] HE K., SUn J., TANG X.: Guided image filtering. In ECCV 2010. Springer, 2010, pp. 1-14. 4

[KSE*03] Kwatra V., SchÖDl A., EsSa I., TURK G., BoBICK A.: Graphcut textures: image and video synthesis using graph cuts. In TOG (2003), vol. 22, ACM, pp. 277-286. 8

[LEN10] Lalonde J.-F., EFros A. A., Narasimhan S. G.: Detecting ground shadows in outdoor consumer photographs. In ECCV 2010. Springer, 2010, pp. 322-335. 2

[LG08] LiU F., GLeicher M.: Texture-consistent shadow removal. In ECCV 2008. Springer, 2008, pp. 437-450. 2, 3

[LLW08] LEVIN A., Lischinski D., Weiss Y.: A closed-form solution to natural image matting. PAMI, IEEE Transactions on 30, 2 (2008), 228-242. 8

[MM96] Manjunath B. S., MA W.-Y.: Texture features for browsing and retrieval of image data. PAMI, IEEE Transactions on 18, 8 (1996), 837-842. 5

[MTC07] Mohan A., Tumblin J., Choudhury P.: Editing soft shadows in a digital photograph. Computer Graphics and Applications, IEEE 27, 2 (2007), 23-31. 3

[Ots75] OTsu N.: A threshold selection method from gray-level histograms. Automatica 11, 285-296 (1975), 23-27. 1, 4

[RAGS01] REINHARD E., AdHIKhMin M., Gooch B., SHIRLEY P.: Color transfer between images. Computer Graphics and Applications, IEEE 21, 5 (2001), 34-41. 3, 6

[SL08] ShOR Y., LischinsKi D.: The shadow meets the mask: Pyramid-based shadow removal. In $C G F$ (2008), vol. 27, pp. 577-586. 1, 2, 3, 4, 6, 7, 8, 9, 10

[TM98] Tomasi C., MANDUCHI R.: Bilateral filtering for gray and color images. In Computer Vision, 1998. Sixth International Conference on (1998), IEEE, pp. 839-846. 3

[Tsa06] TsAI V. J.: A comparative study on shadow compensation of color aerial images in invariant color models. Geoscience and Remote Sensing, IEEE Transactions on 44, 6 (2006), 16611671. 3

[WT05] WU T.-P., TANG C.-K.: A bayesian approach for shadow extraction from a single image. In ICCV 2005 (2005), vol. 1, IEEE, pp. 480-487. 3

[WTBS07] Wu T.-P., TANG C.-K., Brown M. S., Shum H.Y.: Natural shadow matting. TOG 26, 2 (2007), 8. 1, 2, 3

[XG12] XIAO C., GAN J.: Fast image dehazing using guided joint bilateral filter. The Visual Computer 28, 6-8 (2012), 713721. 9

[XSXM13] Xiao C.-X., She R.-Y., XiaO D.-L., Ma K.-L.: Fast shadow removal using adaptive multi-scale illumination transfer. In Computer Graphics Forum (2013). 2, 3, 4, 6

[ZSMT10] Zhu J., Samuel K. G., Masood S. Z., Tappen M. F.: Learning to recognize shadows in monochromatic natural images. In CVPR 2010 (2010), pp. 223-230. 2 